\title{
Dark-Bright Soliton Bound States in a Microresonator
}

\author{
Shuangyou Zhang, ${ }^{1}$ Toby Bi®, ${ }^{1,2}$ George N. Ghalanos@ ${ }^{1,3}$ Niall P. Moroney, ${ }^{1,3}$ \\ Leonardo Del Bino ${ }^{1}{ }^{1}$, and Pascal Del'Haye ${ }^{1,2, *}$ \\ ${ }^{1}$ Max Planck Institute for the Science of Light, 91058 Erlangen, Germany \\ ${ }^{2}$ Department of Physics, Friedrich Alexander University Erlangen-Nuremberg, 91058 Erlangen, Germany \\ ${ }^{3}$ Blackett Laboratory, Imperial College London, SW7 2AZ London, United Kingdom
}

(Received 23 July 2021; accepted 1 December 2021; published 18 January 2022)

\begin{abstract}
Dissipative Kerr solitons in microresonators have facilitated the development of fully coherent, chipscale frequency combs. In addition, dark soliton pulses have been observed in microresonators in the normal dispersion regime. Here, we report bound states of mutually trapped dark-bright soliton pairs in a microresonator. The soliton pairs are generated seeding two modes with opposite dispersion but with similar group velocities. One laser operating in the anomalous dispersion regime generates a bright soliton microcomb, while the other laser in the normal dispersion regime creates a dark soliton via Kerr-induced cross-phase modulation with the bright soliton. Numerical simulations agree well with experimental results and reveal a novel mechanism to generate dark soliton pulses. The trapping of dark and bright solitons can lead to light states with the intriguing property of constant output power while spectrally resembling a frequency comb. These results can be of interest for telecommunication systems, frequency comb applications, and ultrafast optics.
\end{abstract}

DOI: 10.1103/PhysRevLett.128.033901

Optical frequency combs generated in monolithic high$Q$ microresonators have attracted significant research interest [1-4]. Recently, the discovery of dissipative Kerr solitons in microresonators has been demonstrated as a source for low-noise and broadband frequency combs [5,6]. Rich nonlinear dynamics in microresonators has been revealed in the past decade, including breather solitons $[7,8]$, soliton crystals $[9,10]$, Stokes solitons [11], Pockels solitons [12], laser cavity solitons [13], and dark solitons $[14,15]$. In terms of applications, soliton microcombs have already been successfully used for optical frequency synthesizers [16], astronomy [17,18], optical coherent communications [19,20], laser-based light detection and ranging [21-23], and dual-comb spectroscopy [24,25].

Group velocity dispersion of microresonators plays a critical role in microcomb formation. Bright soliton generation in microresonators requires anomalous dispersion at the pump wavelengths while dark solitons can be observed when pumping in the normal dispersion regime. Recent research theoretically predicted the coexistence of bright and dark solitons in the regimes of normal [26], zero [27], and anomalous dispersion [28], when taking account of

Published by the American Physical Society under the terms of the Creative Commons Attribution 4.0 International license. Further distribution of this work must maintain attribution to the author(s) and the published article's title, journal citation, and DOI. Open access publication funded by the Max Planck Society. higher-order dispersion. In addition, bichromatic pumping of microresonators has been studied for thresholdless microcomb generation and stabilization of the repetition rate [29-34]. More recently, bichromatic pumping has been demonstrated for the simultaneous generation of orthogonally polarized microcombs $[35,36]$ and spectral extension of microcombs [37]. The generation of dark-bright soliton pairs via cross-phase modulation (XPM) has been studied since the 1980s [38,39] in mode-locked lasers [40,41] and optical parametric oscillators [42]. However, so far there are no reports of their generation in microresonators. Recently, it has been theoretically predicted that photonic dimers consisting of two strongly coupled microresonators support synchronized states with a bright soliton in one resonator and a dark soliton in the second resonator [43].

Here, we propose and experimentally demonstrate the generation of bound states of dark and bright soliton pairs in a single microresonator, as illustrated in Fig. 1, two seed lasers jointly pump a microresonator. One laser $\left(\lambda_{1}\right.$, red $)$ in the anomalous dispersion regime (referred to as primary pump) generates a bright soliton microcomb. The second laser ( $\lambda_{2}$, blue, referred to as auxiliary pump) seeds the microresonator in the normal dispersion regime and passively forms dark soliton pulses through Kerr-induced XPM with the bright soliton. The dark soliton is trapped by the bright soliton in the time domain, resulting in a copropagating pair of bright and dark solitons. A prerequisite for the generation of dark-bright soliton pairs is a similar free spectral range (FSR) in the spectral regions of both pump lasers. Numerical simulations confirm the 


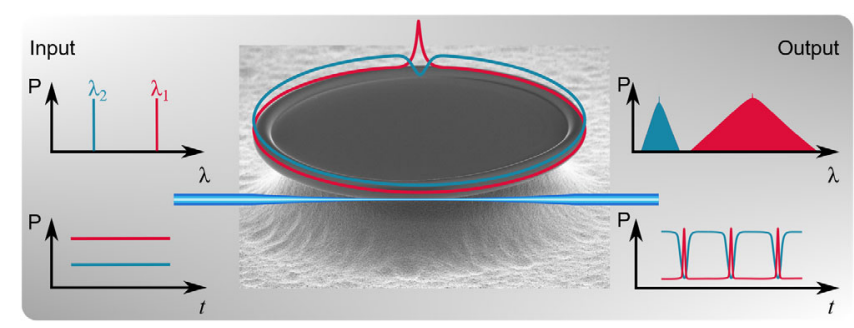

FIG. 1. Scheme for dark-bright soliton pair generation in a microresonator. One seed laser $\left(\lambda_{1}\right.$, red) operates in the anomalous dispersion regime, generating a bright soliton microcomb, while a second laser $\left(\lambda_{2}\right.$, blue) excites a dark soliton in the normal dispersion regime. Both lasers operate in spectral regions with similar free spectral ranges. The dark soliton is synchronized with the bright soliton in time domain, mediated by cross-phase modulation. Inset: Scanning electron microscope image of a silica microtoroid resonator used in the experiments.

experimental results and reveal a novel mechanism to generate dark soliton pulses. To our knowledge, this is the first demonstration of simultaneous generation and trapping of bright and dark cavity solitons in passive resonators. The demonstrated technique could be useful for optical signal processing and optical telecommunication with better stability in the presence of noise and nonlinearities [44].

Considering a passive ring resonator made of a dispersive medium with Kerr nonlinearity, the resonance frequencies of a mode family can be described as

$$
\omega_{*, \mu}=\omega_{*, 0}+D_{*, 1} \mu+\frac{D_{*, 2}}{2 !} \mu^{2}+\frac{D_{*, 3}}{3 !} \mu^{3},
$$

where $\mu$ is the mode number offset from the pump mode at $\mu=0 . \omega_{*, \mu}$ is the resonance frequency of that mode, “*” refers to either the primary (" $p$ ") or auxiliary (" $a$ ") mode family, $D_{*, 1} / 2 \pi$ is the FSR of the resonator at the primary $\left(D_{p, 1} / 2 \pi\right)$ or auxiliary $\left(D_{a, 1} / 2 \pi\right)$ pump mode, and $D_{*, 2}$, $D_{*, 3}$ are coefficients of second- and third-order dispersion, respectively. The two microresonator modes for the primary and auxiliary pumps can be either from the same mode family or different mode families [37]. Figure 2(a) shows the normalized FSR mismatch $\gamma(\mu)=\left[D_{a, 1}-\right.$ $\left.D_{p, 1}(\mu)\right] / \Delta \omega_{0}$ (blue curve) as a function of the mode number $\mu$. The FSR mismatch $\gamma(\mu)$ is normalized to the full-width half-maximum (FWHM) of the resonance $\Delta \omega_{0}$. Microresonators typically exhibit different dispersion over a broad spectral range. For example, silica microresonators have normal dispersion (FSR decreases with optical frequency) at short wavelengths and anomalous dispersion (FSR increases with optical frequency) at long wavelengths. As a result, it is possible to find two optical modes at different wavelengths, and in opposite dispersion regimes with a similar FSR. The simulation (blue curve) plotted in Fig. 2(a) shows $\gamma(\mu)$ crossing zero when the

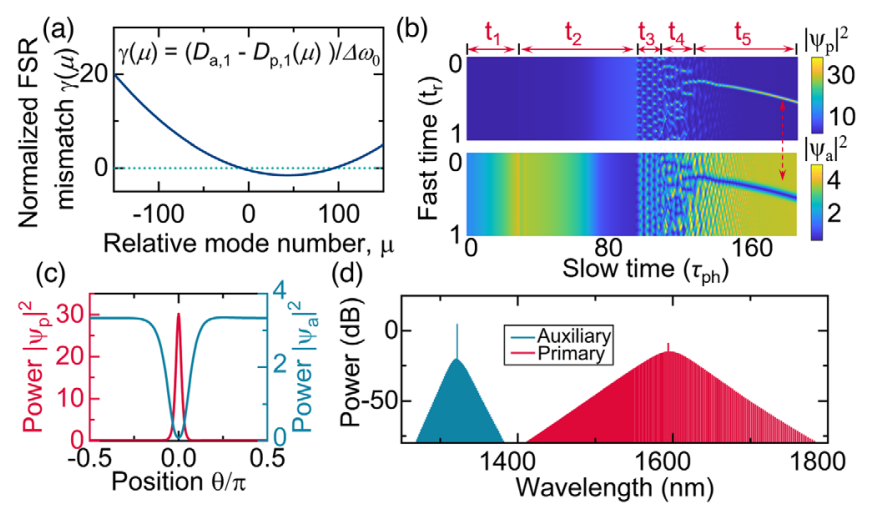

FIG. 2. Numerical simulation of dark-bright solitons pairs in a microresonator. (a) Normalized group velocity mismatch $\gamma(\mu)$ (blue, solid line) between the auxiliary pump mode and the primary modes as a function of the mode number $\mu$. (b) Temporal waveform evolution of the primary bright soliton (upper panel) and auxiliary dark soliton (lower panel) when the primary laser seeds an optical mode at $\mu=-8$ (where the FSR matches the FSR around the auxiliary mode). $\tau_{\mathrm{ph}}$ : photon lifetime; $t_{r}$ : roundtrip time. $\left|\psi_{a}\right|^{2}$ and $\left|\psi_{p}\right|^{2}$ are the corresponding intracavity powers. (c) Temporal waveforms of a bright soliton (red, left axis) and its induced dark soliton (blue, right axis) at the position marked with the red dashed arrow in (b). (d) Simulated intracavity optical spectrum corresponding to the temporal waveforms shown in (c).

relative mode number is around $-8(\gamma \approx-0.02$ for $\mu=-8)$. Thus, the group velocity of the primary pump mode at $\mu=-8$ is almost identical to that for the auxiliary pump mode.

Here, we perform numerical simulations based on two simultaneous, generalized Lugiato-Lefever equations [45-47], with additional XPM terms allowing interaction between primary and auxiliary fields and considering the group velocity mismatch between the primary and auxiliary pump modes [35-37,48,49]. From Fig. 2(a), the primary pump mode is selected at $\mu=-8$ to match the group velocity of the auxiliary mode. Further details on numerical simulation parameters are outlined in the Supplemental Material [50]. Figure 2(b) shows the generation and evolution of a dark-bright soliton pair. To match the simulation with experimental techniques, the auxiliary pump frequency is initially blue detuned with respect to its resonance $\left(t_{1}\right)$. Then the primary pump's frequency is scanned from the blue- to red-detuned side of its resonance until accessing stable soliton states $\left(t_{2}\right.$ to $\left.t_{5}\right)$. The Kerrinduced resonance shift resulting from the rising intracavity primary power causes the decrease of the intracavity auxiliary power $\left(t_{2}\right)$. When further tuning the primary laser into its resonance, the intracavity power will surpass the hyperparametric oscillation threshold power, and the primary field starts to generate Turing rolls $\left(t_{3}\right)$ [51]. Simultaneously, the auxiliary field also generates Turing rolls through Kerr XPM with the primary Turing rolls, 
however, they are out of phase with each other (see Fig. S1 in Supplemental Material for details [50]). When continuing to tune the primary laser into its resonance, the primary comb will enter a chaotic regime with low coherence $\left(t_{4}\right)$, where both the intracavity primary and auxiliary waveform are unstable. Once the primary laser enters the red-detuned side of the resonance $\left(t_{5}\right)$, a bright primary soliton is formed. Strikingly, a dark soliton is simultaneously generated via the XPM interaction between the intracavity auxiliary field and the bright soliton. The dark soliton is stable and propagates at the same velocity as the bright soliton. Note that the generation of the dark soliton is based on a nondegenerate thresholdless fourwave-mixing process [29]. Whenever the primary bright soliton is accessed, a dark soliton will be generated and synchronized with the bright soliton without a threshold power. See Supplemental Material [50] for an animation of the dark-bright soliton pair formation.

Figure 2(c) shows the simulated intracavity temporal waveforms of a single-soliton primary microcomb (red, left axis) and its induced auxiliary dark soliton pulse (blue, right axis). The soliton region is marked with a dashed arrow in Fig. 2(b). The auxiliary dark soliton is trapped at the maximum of the bright pulse. The intracavity optical spectra corresponding to the temporal waveforms of Fig. 2(c) are shown in Fig. 2(d). Compared with optical spectra of conventional microresonator dark solitons in the normal-dispersion regime [14,15], the spectral envelope shown in Fig. 2(d) does not exhibit "cat-ear" peaks neighboring the auxiliary pump, which arise through the interlocking of switching waves that connect the homogenous steady states of the bistable cavity system [26]. Thus, in contrast to conventional dark solitons, we do not observe small intensity oscillations close to the dark soliton minima. Note that when the primary microcomb is in a multisoliton state, the resonator can also generate multiple dark-bright soliton pairs (see Fig. S2 in the Supplemental Material [50]).

To investigate the influence of the group velocity mismatch on the dark soliton, we performed a number of simulations by changing the mode number that is pumped by the primary laser, while keeping the auxiliary laser seeding the same optical mode as in Fig. 2. Figure 3 shows the simulation results for dark-bright soliton pairs at different group velocity mismatch when the primary soliton microcomb is in a single-soliton state. Figure 3(a) illustrates the simulated intracavity optical spectrum of the auxiliary dark soliton when the primary pump mode is at $\mu=-1$ with $\gamma=-0.41$. The corresponding temporal waveform of the dark soliton (blue) is shown in Fig. 3(b) with the red trace depicting the temporal waveform of the primary bright soliton. The spectra of the dark-bright soliton states can be found in Fig. S3 in the Supplemental Material [50]. Figures 3(c) and 3(d) show the simulation results when the primary pump mode is at
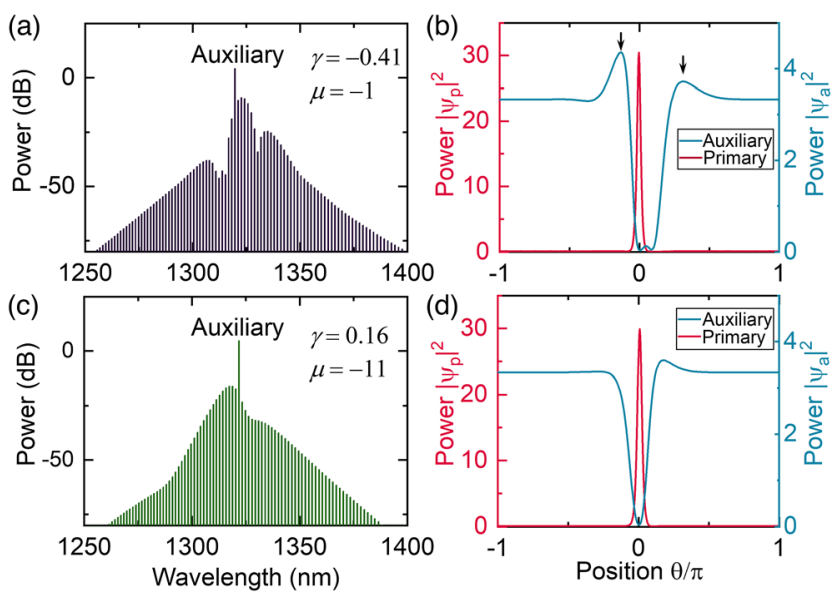

FIG. 3. Dark-bright soliton pairs at different group velocity mismatch. (a) Simulated optical spectrum and its corresponding temporal waveform (b) of the auxiliary dark soliton while the primary soliton microcomb is in a single-soliton state seeded at mode number $\mu=-1$, with FSR mismatch $\gamma=-0.41$. (c) Simulated optical spectrum and its corresponding temporal waveform (d) of the auxiliary dark soliton when the primary soliton microcomb is seeded at $\mu=-11$ with $\gamma=0.16$.

$\mu=-11$ with $\gamma=0.16$. Interestingly, the spectral and temporal profiles of the dark soliton change as seen in Figs. 3(c) and 3(d). Compared with Fig. 2(d), the spectra in Figs. 3(a) and 3(c) are more asymmetrical and irregular when the value of $\gamma$ is further from zero. The peak of the envelope of the auxiliary microcomb is shifted from the red [Fig. 3(a)] to the blue side [Fig. 3(c)] of the auxiliary pump frequency when the primary pump mode is changed from $\mu=-1$ to -11 , which corresponds to a change of $\gamma$ from negative to positive. This asymmetry is also present in the time domain [as shown in Figs. 3(b) and 3(d)] where the relative amplitudes of the sidelobes-peaks of the dark soliton are changing when changing the sign of $\gamma$. Moreover, it is observed that the duration of the dark soliton increases when the absolute value of $\gamma$ increases. These results show that the group velocity mismatch $\gamma$ plays an important role in the temporal shape of the dark soliton. Additional analysis of dark-bright soliton pairs is presented in the Supplemental Material [50], including their existence range (Fig. S4), stability charts (Fig. S5), and the observation of Arnold tongues [52,53] (Fig. S6).

The numerical predictions are verified with experiments using fused silica microtoroid resonators. Figure 4(a) shows the schematic of the experimental setup. Two external cavity diode lasers (ECDLs) are used for bichromatic pumping of a resonator. The primary laser at $1.5-\mu \mathrm{m}$ wavelength generates a bright soliton microcomb, where the overall group velocity dispersion is anomalous. The auxiliary laser at $1.3 \mu \mathrm{m}$ passively stabilizes the circulating optical power to assist bright soliton generation for the primary pump $[54,55]$ and simultaneously generates a dark soliton pulse. A $235-\mu$ m-diameter fused silica microtoroid 


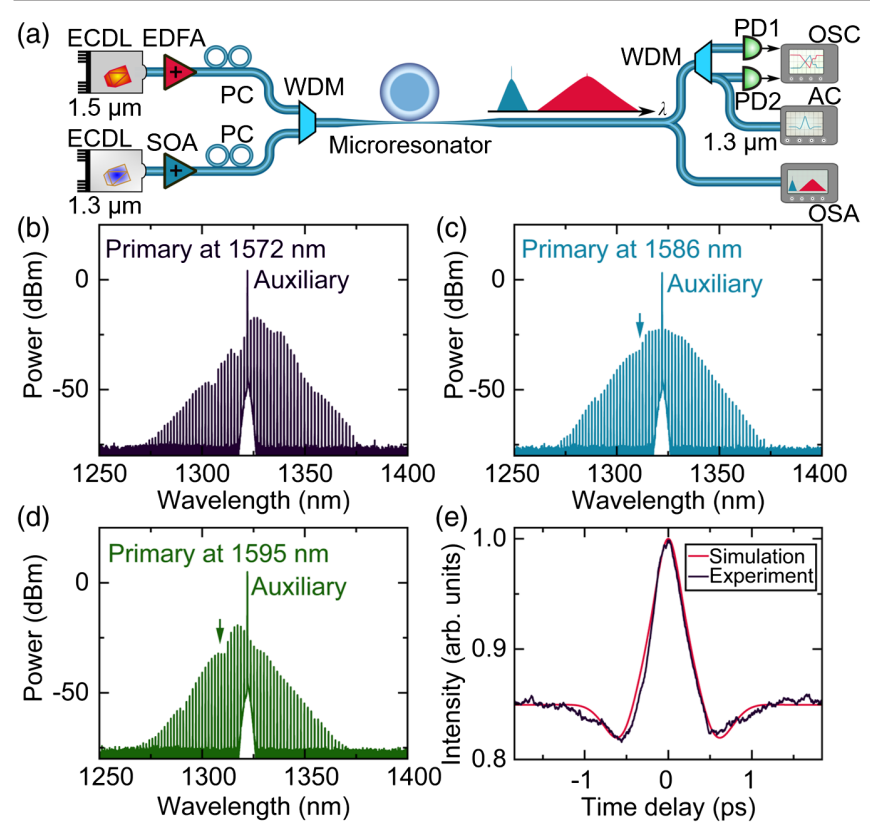

FIG. 4. Experimental demonstration of dark-bright soliton pairs. (a) Setup for the dark-bright soliton pair generation. The $1.5-\mu \mathrm{m}$ primary pump laser is used to generate a bright soliton, while the $1.3-\mu \mathrm{m}$ auxiliary laser thermally stabilizes the resonator and at the same time generates a dark soliton via XPM with the bright soliton. ECDL: external cavity diode laser; EDFA: erbiumdoped fiber amplifier; SOA: semiconductor optical amplifier; WDM: wavelength division multiplexer; PC: polarization controller; PD: photodetector; AC: autocorrelator; OSA: optical spectrum analyzer; OSC: oscilloscope. (b)-(d) Measured spectra of the dark soliton frequency combs at different central wavelengths of the bright soliton. The bright soliton is seeded at $1572 \mathrm{~nm}$ in (b), $1586 \mathrm{~nm}$ in (c), and $1595 \mathrm{~nm}$ in (d). The arrows in (c) and (d) indicate a spectral dip induced by mode crossings. (e) Autocorrelation trace (black) of the dark soliton with the spectrum shown in (b) together with a calculated autocorrelation trace (red) of the dark soliton shown in the blue trace in Fig. 3(b).

is used in the experiments with an FSR of $274 \mathrm{GHz}$. This particular microresonator is fabricated from a silicon wafer with a 6- $\mu$ m-layer of silicon dioxide $\left(\mathrm{SiO}_{2}\right)$ [56]. The two pump lasers are combined with a wavelength division multiplexer (WDM) and evanescently coupled into the microresonator via a tapered optical fiber. Two fiber polarization controllers (PCs) are used to match the polarization of the two lasers. At the resonator output, part of the light is monitored by an optical spectrum analyzer (OSA). A second part of the light is separated by another WDM to monitor the pump lasers separately on two photodiodes (PD1 and PD2). An intensity autocorrelator is used to measure the autocorrelation traces of the dark soliton pulses. A fiber Bragg grating filter suppresses the residual auxiliary pump power before the autocorrelator. The primary mode family is carefully selected so that it not only supports the formation of a bright soliton, but also has a selection of modes with FSRs that match the group velocity of the auxiliary mode. In the experiments, the $1.5-\mu \mathrm{m}$ primary pump laser is widely tuned from 1560 to $1600 \mathrm{~nm}$. Within this range, each mode from the primary mode family supports the formation of a bright soliton. Figure 4(b) shows the dark soliton microcomb spectrum that is generated by the interplay of the primary bright soliton at $1572 \mathrm{~nm}$ seeded by $200 \mathrm{~mW}$, together with $160 \mathrm{~mW}$ of auxiliary pump light at $1322 \mathrm{~nm}$. See Fig. S7 in the Supplemental Material [50] for the full spectrum including a spectrum of the single-soliton primary microcomb. We note that the dark soliton spectrum exhibits an irregular shape that agrees well with the simulated comb spectrum shown in Fig. 3(a). In addition to the similar spectral envelope, the spectral peak of the auxiliary frequency comb also appears on the red side of the auxiliary pump, which is consistent with the simulation in Fig. 3(a). To qualitatively explore the impact of the group velocity mismatch between the primary and auxiliary pump mode on the auxiliary microcomb, the primary pump wavelength is changed to different modes between 1572 and $1595 \mathrm{~nm}$ while the auxiliary pump laser was held at a fixed mode at $1322 \mathrm{~nm}$. As shown in Fig. 4(c), the auxiliary microcomb assumes a symmetric shape around its pump when the primary microcomb is in a single-soliton state at $1586 \mathrm{~nm}$. This is consistent with the simulated spectra shown in Fig 2(d). In addition, the symmetric spectrum suggests that the group velocity mismatch between the auxiliary mode and primary pump mode at $1586 \mathrm{~nm}$ is close to zero. The primary pump wavelength was further tuned to $1595 \mathrm{~nm}$, which leads to the generation of an auxiliary frequency comb with a similar envelope as the one in Fig. 3(c) with a positive group velocity mismatch. The corresponding dark soliton spectrum is shown in Fig. 4(d) and exhibits a combenvelope peak shifted to the blue side of the auxiliary pump frequency, in agreement with the simulation in Fig. 3(c). The spectral defects (marked with arrows) that are present in both Figs 4(c) and 4(d), are most likely generated by mode crossings with other mode families [57]. See Fig. S8 in the Supplemental Material [50] for measurements of spectra with multiple dark-bright soliton pairs.

The auxiliary combs presented in Fig. 4 agree well with the simulations in Figs. 2 and 3 in terms of their spectral envelopes and the dependence of the spectral peak on the group velocity mismatch. When the primary pump wavelength is tuned from short wavelength $(1572 \mathrm{~nm})$ to long wavelength $(1595 \mathrm{~nm})$, the group velocity mismatch changes from negative to positive, crossing zero at around $1586 \mathrm{~nm}$. This is similar to the simulations in Fig. 3: when changing the sign of the group velocity mismatch from negative to positive, the spectral peak is shifted from the red to blue side of the auxiliary pump.

To further confirm our findings, we measure the autocorrelation traces of the generated auxiliary dark solitons. Figure 4(e) shows a normalized autocorrelation trace (black) of the dark soliton with a corresponding spectrum 
in Fig. 4(b). For comparison, a simulated autocorrelation trace (red) of the dark soliton [blue trace shown in Fig. 3(b)] is overlaid. Both the experimental measurement and the simulation contain two dips on the sides of the autocorrelation peak, which are associated with the peaks shown in Fig. 3(b) (marked with arrows). The high background intensity of the autocorrelation trace is a result of the relatively short pulse duration of the dark soliton of $\sim 500 \mathrm{fs}$.

Conventional dark soliton formation in normaldispersion microresonators requires the aid of mode interactions, which can be realized via modal coupling between different mode families in an overmoded microresonator $[14,15]$ or via mode interaction from an additional coupled microresonator [58]. In contrast, the demonstrated method here generates dark solitons via the Kerr XPM with bright solitons, rather than the assistance of mode crossings. By pumping an auxiliary mode with similar FSR to the primary mode, the local normal dispersion of the auxiliary mode is still preserved, and not modified via the synchronization of the bright and dark soliton, hence, enabling the generation of dark solitons [37]. The auxiliary pump mode could also be selected from an orthogonally polarized mode family with respect to the primary pump mode, which enables the generation of orthogonally polarized darkbright pulse pairs.

In summary, we proposed and experimentally demonstrated the generation of bound states of dark-bright solitons in a single microresonator with two seed lasers: one laser operating in the anomalous dispersion regime generates a bright soliton, while the second laser generates a dark soliton in the normal dispersion regime, mediated by XPM with the bright soliton. Both solitons share a similar group velocity. Operating the primary microcomb in a multisoliton state, this technique can generate multiple dark-bright soliton pairs. The mutually trapped dark-bright soliton pairs enable waveforms with nearly constant output power in the time domain, which makes them less susceptible to perturbations and noise in optical systems. In particular, comb sources with constant output power could enable new ways of optical amplification that avoid limitations imposed by high peak powers, given a sufficiently broadband gain medium. Besides the fascinating soliton dynamics, such a system could also be of interest for the generation of broadband optical frequency comb sources that span across different dispersion regimes. This could be beneficial for applications ranging from precision spectroscopy to laser ranging and optical telecommunication systems. The presented results could also lead to new insights in the understanding of complex soliton dynamics in ultrafast nonlinear optics.

This work is supported by the European Union's H2020 ERC Starting Grant No. "CounterLight" 756966; H2020 Marie Sklodowska-Curie COFUND "Multiply" 713694; Marie Curie Innovative Training Network "Microcombs"
812818; Max Planck Society, and the Engineering and Physical Sciences Research Council (EPSRC) via the CDTs for Applied Photonics, Controlled Quantum Dynamics, and Quantum Systems Engineering.

*pascal.delhaye@mpl.mpg.de

[1] P. Del'Haye, A. Schliesser, O. Arcizet, T. Wilken, R. Holzwarth, and T. J. Kippenberg, Nature (London) 450 , 1214 (2007).

[2] T. J. Kippenberg, R. Holzwarth, and S. A. Diddams, Science 332, 555 (2011).

[3] B. Stern, X. Ji, Y. Okawachi, A. L. Gaeta, and M. Lipson, Nature (London) 562, 401 (2018).

[4] C. Xiang et al., Science 373, 99 (2021).

[5] T. Herr, V. Brasch, J. D. Jost, C. Y. Wang, N. M. Kondratiev, M. L. Gorodetsky, and T. J. Kippenberg, Nat. Photonics 8 , 145 (2014).

[6] T. J. Kippenberg, A. L. Gaeta, M. Lipson, and M. L. Gorodetsky, Science 361, eaan8083 (2018).

[7] M. Yu, J. K. Jang, Y. Okawachi, A. G. Griffith, K. Luke, S. A. Miller, X. Ji, M. Lipson, and A. L. Gaeta, Nat. Commun. 8, 14569 (2017).

[8] E. Lucas, M. Karpov, H. Guo, M. L. Gorodetsky, and T. J. Kippenberg, Nat. Commun. 8, 736 (2017).

[9] D. C. Cole, E. S. Lamb, P. Del'Haye, S. A. Diddams, and S. B. Papp, Nat. Photonics 11, 671 (2017).

[10] M. Karpov, M. H. P. Pfeiffer, H. Guo, W. Weng, J. Liu, and T. J. Kippenberg, Nat. Phys. 15, 1071 (2019).

[11] Q.-F. Yang, X. Yi, K. Y. Yang, and K. Vahala, Nat. Phys. 13, 53 (2017).

[12] A. W. Bruch, X. Liu, Z. Gong, J. B. Surya, M. Li, C.-L. Zou, and H. X. Tang, Nat. Photonics 15, 21 (2021).

[13] H. Bao et al., Nat. Photonics 13, 384 (2019).

[14] X. Xue, Y. Xuan, Y. Liu, P.-H. Wang, S. Chen, J. Wang, D. E. Leaird, M. Qi, and A. M. Weiner, Nat. Photonics 9, 594 (2015).

[15] E. Nazemosadat, A. Fülöp, Ó. B. Helgason, P.-H. Wang, Y. Xuan, D. E. Leaird, M. Qi, E. Silvestre, A. M. Weiner, and Victor Torres-Company, Phys. Rev. A 103, 013513 (2021).

[16] D. T. Spencer et al., Nature (London) 557, 81 (2018).

[17] E. Obrzud et al., Nat. Photonics 13, 31 (2019).

[18] M.-G. Suh et al., Nat. Photonics 13, 25 (2019).

[19] P. Marin-Palomo et al., Nature (London) 546, 274 (2017).

[20] A. Fülöp, M. Mazur, A. Lorences-Riesgo, Ó. B. Helgason, P.-H. Wang, Y. Xuan, D. E. Leaird, M. Qi, P. A. Andrekson, A. M. Weiner, and V. Torres-Company, Nat. Commun. 9, 1598 (2018).

[21] M.-G. Suh and K. J. Vahala, Science 359, 884 (2018).

[22] P. Trocha et al., Science 359, 887 (2018).

[23] N. Kuse and M.E. Fermann, APL Photonics 4, 106105 (2019).

[24] M.-G. Suh, Q.-F. Yang, K. Y. Yang, X. Yi, and K. J. Vahala, Science 354, 600 (2016).

[25] A. Dutt, C. Joshi, X. Ji, J. Cardenas, Y. Okawachi, K. Luke, A. L. Gaeta, and M. Lipson, Sci. Adv. 4, e1701858 (2018).

[26] P. Parra-Rivas, D. Gomila, and L. Gelens, Phys. Rev. A 95, 053863 (2017). 
[27] J. H. Talla Mbé, C. Milián, and Y. K. Chembo, Eur. Phys. J. D 71, 196 (2017).

[28] J. H. T. Mbé and Y. K. Chembo, J. Opt. Soc. Am. B 37, A69 (2020).

[29] D. V. Strekalov and N. Yu, Phys. Rev. A 79, 041805(R) (2009).

[30] T. Hansson and S. Wabnitz, Phys. Rev. A 90, 013811 (2014).

[31] H. Taheri, A. B. Matsko, and L. Maleki, Eur. Phys. J. D 71, 153 (2017).

[32] C. Bao et al., Opt. Lett. 42, 595 (2017).

[33] W. Wang et al., Sci. Rep. 6, 28501 (2016).

[34] R. J. Weiblen and I. Vurgaftman, Opt. Express 27, 4238 (2019).

[35] C. Bao et al., Opt. Lett. 44, 1472 (2019).

[36] R. Suzuki, S. Fujii, A. Hori, and T. Tanabe, IEEE Photonics J. 11, 1 (2019).

[37] S. Zhang, J. M. Silver, T. Bi, and P. Del'Haye, Nat. Commun. 11, 6384 (2020).

[38] S. Trillo, S. Wabnitz, E. M. Wright, and G. I. Stegeman, Opt. Lett. 13, 871 (1988).

[39] V. V. Afanasjev, E. M. Dianov, and V. N. Serkin, IEEE J. Quantum Electron. 25, 2656 (1989).

[40] Q. Ning, S. Wang, A. Luo, Z. Lin, Z. Luo, and W. Xu, IEEE Photonics J. 4, 1647 (2012).

[41] G. Shao, Y. Song, L. Zhao, D. Shen, and D. Tang, Opt. Express 23, 26252 (2015).

[42] M. Jankowski, A. Marandi, C. R. Phillips, R. Hamerly, K. A. Ingold, R. L. Byer, and M. M. Fejer, Phys. Rev. Lett. 120, 053904 (2018).

[43] K. Komagata, A. Tusnin, J. Riemensberger, M. Churaev, H. Guo, A. Tikan, and T. J. Kippenberg, Commun. Phys. 4, 159 (2021).

[44] Y. S. Kivshar and B. Luther-Davies, Phys. Rep. 298, 81 (1998).
[45] L. A. Lugiato and R. Lefever, Phys. Rev. Lett. 58, 2209 (1987).

[46] S. Coen, H. G. Randle, T. Sylvestre, and M. Erkintalo, Opt. Lett. 38, 37 (2013).

[47] Y. K. Chembo and C. R. Menyuk, Phys. Rev. A 87, 053852 (2013).

[48] L. Hill, G.-L. Oppo, M. T. M. Woodley, and P. Del'Haye, Phys. Rev. A 101, 013823 (2020).

[49] C. Godey, I. V. Balakireva, A. Coillet, and Y. K. Chembo, Phys. Rev. A 89, 063814 (2014).

[50] See Supplemental Material at http://link.aps.org/supplemental/ 10.1103/PhysRevLett.128.033901 for simulation parameters, additional analysis, and full measurement spectra.

[51] A. Coillet, I. Balakireva, R. Henriet, K. Saleh, L. Larger, J. M. Dudley, C. R. Menyuk, and Y. K. Chembo, IEEE Photonics J. 5, 6100409 (2013).

[52] J. K. Jang, X. Ji, C. Joshi, Y. Okawachi, M. Lipson, and A. L. Gaeta, Phys. Rev. Lett. 123, 153901 (2019).

[53] A. Pikovsky, M. Rosenblum, and J. Kurths, Synchronization: A Universal Concept in Nonlinear Sciences (Cambridge University Press, Cambridge, England, 2001).

[54] S. Zhang, J. M. Silver, L. D. Bino, F. Copie, M. T. M. Woodley, G. N. Ghalanos, A. Ø. Svela, N. Moroney, and P. Del'Haye, Optica 6, 206 (2019).

[55] H. Zhou, Y. Geng, W. Cui, S.-W. Huang, Q. Zhou, K. Qiu, and C. Wei Wong, Light Sci. Appl. 8, 50 (2019).

[56] S. Zhang, J. M. Silver, J. M. Silver, X. Shang, L. D. Bino, L. D. Bino, N. M. Ridler, and P. Del'Haye, Opt. Express 27, 35257 (2019).

[57] T. Herr, V. Brasch, J. D. Jost, I. Mirgorodskiy, G. Lihachev, M. L. Gorodetsky, and T. J. Kippenberg, Phys. Rev. Lett. 113, 123901 (2014).

[58] X. Xue, Y. Xuan, P.-H. Wang, Y. Liu, D. E. Leaird, M. Qi, and A. M. Weiner, Laser Photonics Rev. 9, L23 (2015). 